\title{
Glycoproteomic studies of IgE from a novel hyper IgE syndrome linked to PGM3 mutation
}

\author{
Gang Wu ${ }^{1,2}$ • Paul G. Hitchen ${ }^{1}$ Maria Panico ${ }^{1} \cdot$ Simon J. North $^{1}$ • \\ Mohamed-Ridha Barbouche ${ }^{3}$ • Daniel Binet ${ }^{4} \cdot$ Howard R. Morris ${ }^{1,4}$ • Anne Dell ${ }^{1}$ • \\ Stuart M. Haslam ${ }^{1,5}$
}

Received: 10 August 2015 /Revised: 20 October 2015 / Accepted: 4 November 2015 /Published online: 19 December 2015

(C) The Author(s) 2015. This article is published with open access at Springerlink.com

\begin{abstract}
Glycans serve as important regulators of antibody activities and half-lives. IgE is the most heavily glycosylated antibody, but in comparison to other antibodies little is known about its glycan structure function relationships. We therefore describe the site specific IgE glycosylation from a patient with a novel hyper IgE syndrome linked to mutations in PGM3, which is an enzyme involved in synthesizing UDP-GlcNAc, a sugar donor widely required for glycosylation. A two-step method was developed to prepare two IgE samples from less than $1 \mathrm{~mL}$ of serum collected from a patient with $P G M 3 \mathrm{mu}-$ tation and a patient with atopic dermatitis as a control subject. Then, a glycoproteomic strategy was used to study the sitespecific glycosylation. No glycosylation was found at Asn264, whilst high mannose glycans were only detected at Asn275, tri-antennary glycans were exclusively observed at Asn99 and Asn252, and non-fucosylated complex glycans
\end{abstract}

Electronic supplementary material The online version of this article (doi:10.1007/s10719-015-9638-y) contains supplementary material, which is available to authorized users.

Stuart M. Haslam

s.haslam@imperial.ac.uk

1 Department of Life Sciences, Imperial College London, South Kensington Campus, London SW7 2AZ, UK

2 Division of Cell Signalling and Immunology, School of Life Sciences, University of Dundee, Dundee DD1 5EH, UK

3 Laboratory of Immunopathology, Vaccinology and Molecular Genetics, Pasteur Institute of Tunis and University Tunis El Manar, Tunis, Tunisia

4 MS-RTC (Mass Spectrometry Research and Training Centre), Suite 3.1 Lido Medical Centre, St. Saviours Road, Jersey JE2 7LA, UK

5 Department of Life Sciences, Faculty of Natural Sciences, Imperial College London, South Kensington Campus, London SW7 2AZ, UK were detected at Asn99. The results showed similar glycosylation profiles between the two IgE samples. These observations, together with previous knowledge of IgE glycosylation, imply that $\operatorname{IgE}$ glycosylation is similarly regulated among healthy control, allergy and PGM3 related hyper IgE syndrome.

Keywords IgE $\cdot$ Glycoproteomics $\cdot$ Hyper IgE Syndrome · Phosphoglucomutase 3

\section{Introduction}

All antibodies are glycosylated and the glycans serve as important regulators for antibody activities [1-6] and half-lives [7, 8]. $\mathrm{IgE}$ is known to play a key role in allergic responses [9]. It is the most heavily glycosylated antibody and contains seven potential N-glycosylation sites at Asn21, 49, 99, 146, 252, 264 and 275 in the heavy chain constant region. However, glycosylation of $\operatorname{IgE}$ is not extensively studied, due to its low concentration in the blood, making it difficult to prepare. In the 1970s, monosaccharide ratios of glycans at each potential glycosylation site on a myeloma IgE were reported [10-12]. High variations of glycosylation among different sites were observed, for example Asn264 was found not to be glycosylated and only Man and GlcNAc residues were detected at Asn275. The other 5 sites were occupied by complex glycans with different levels of fucosylation and sialylation. In 2004, an overall profile of IgE $\mathrm{N}$-glycans from an atopic patient was obtained using HPLC [13], which detected $24 \mathrm{~N}$-glycans including both high mannose glycans and complex glycans. More than $75 \%$ of the glycan pool was sialylated, almost $60 \%$ of glycans were core fucosylated, and $13 \%$ of glycans had bisecting GlcNAc. Recently, a mass spectrometry based glycoproteomic strategy was used to obtain a site specific analysis of IgE Nglycosylation [14]. The results showed for the first time the 
glycan structures at each site on IgE, which was collected from a hyperimmune donor, from the pooled serum of multiple nondiseased donors, and from the pooled serum of 2 patients with IgE myeloma. Consistent with the monosaccharide studies of a myeloma IgE, the data showed Asn264 was not glycosylated and Asn275 was glycosylated exclusively with high mannose glycans.

However, little is known about the glycosylation of $\operatorname{IgE}$ from patients with hyper IgE syndrome (HIES). HIES is a primary immunodeficiency disorder which is characterized by dramatically elevated IgE levels, accompanied by other symptoms such as eczema, recurrent staphylococcal skin infections and pulmonary infections [15]. The disease was first reported in 1966 [16], and its autosomal dominant inheritance pattern was discovered in 1999 [17]. Mutations in two proteins were discovered to be linked to the disease: signal transducer and activator of transcription 3 (STAT3) $[18,19]$ and dedicator of cytokinesis protein 8 (DOCK8) [20, 21]. Recently, a novel hyper IgE syndrome has been discovered $[22,23]$, without mutations in STAT3 or DOCK8. Instead, the disease is linked to mutations in phosphoglucomutase 3 (PGM3). Unlike other phosphoglucomutases, which catalyse the conversion of Glc-6-phosphate and Glc-1-phosphate, PGM3 catalyses the conversion of GlcNAc-6-phosphate and GlcNAc-1-phosphate, producing the precursor for synthesizing UDP-GlcNAc, which is a sugar donor required for glycosylation. Our glycomic studies have already found dramatic changes of N-glycans in Epstein-Barr virus (EBV) treated B cells and neutrophils from these patients [23]. Moreover, our glycomic studies of $\mathrm{IgE}$ showed that the overall profile of Nglycans did not change significantly from a patient with PGM3 mutations and a patient with atopic dermatitis [23]. Both IgE samples had high mannose glycans and complex glycans. Most of the complex glycans were bi-antennary with core fucose and sialic acid. Bisecting GlcNAc was observed in some of the bi-antennary structures. Tri-antennary glycans and truncated glycans were also detected. The relative intensities among these glycans were similar between the two IgE samples. However, it is unknown whether there are site specific changes which could be involved in the elevated $\operatorname{IgE}$ which could affect IgE activities and half-life.

In this study, immunoprecipitation was used to prepare two IgE samples from less than $1 \mathrm{~mL}$ of sera/plasma: one was from a patient with $P G M 3$ mutation, the other was from a patient with atopic dermatitis as a control subject. Then we used glycoproteomic strategies to study the glycans at each potential glycosylation site of the two samples. The results showed there are no significant differences between the two IgE samples. Moreover, our data combined with a recent study [14] show that IgE glycoproteomic spectra are similar among healthy controls, patients with allergy and the patient with HIES caused by $P G M 3$ mutation. These observations imply that, despite alterations occurring in the $\mathrm{N}$-glycome of immune cells from patients with $P G M 3$ mutations, the elevated IgE in allergy and HIES may not be related to glycosylation on the antibody itself.

\section{Materials and methods}

\section{Serum/plasma samples}

Serum/plasma samples were collected from the Centre of Chronic Immunodeficiency (CCI), University Medical Center Freiburg, under human subject protocols approved by local ethics committees at University College London, the University of Freiburg and the Pasteur Institute of Tunis.

\section{IgE enrichment}

IgE was enriched by immunoprecipitation using Pierce ${ }^{\circledR}$ Direct IP Kit (Thermo Scientific, Basingstoke, UK) according to the manufacturer's instructions with some modifications. In order to immobilise an anti-IgE antibody on beads, the Pierce Spin Column was loaded with $30 \mu \mathrm{L}$ AminoLink Plus Coupling resin and the liquid was removed by centrifuging at $1000 \mathrm{~g}$ for $1 \mathrm{~min}$. Then, $300 \mu \mathrm{L} 1 \times$ Coupling Buffer was used to wash the resin twice. After that, $185 \mu \mathrm{L} \mathrm{H}_{2} \mathrm{O}, 15 \mu \mathrm{L}$ $20 \times$ Coupling Buffer, $100 \mu \mathrm{L}$ IgE (4F4): sc-51994, mouse monoclonal antibody raised against $\mathrm{IgE}$ of human origin (Santa Cruz Biotechnology, Heidelberg, Germany) and $4.5 \mu \mathrm{L}$ sodium cyanoborohydride were added onto the resin in the column and incubated at room temperature for $90 \mathrm{~min}$ in a rotator. The liquid was removed from the spin column by centrifuging at $1000 \mathrm{~g}$ for $1 \mathrm{~min}$. The resin was washed twice with $300 \mu \mathrm{L} 1 \times$ Coupling Buffer and $300 \mu \mathrm{L}$ of $1 \times$ Quenching Buffer. Then, $300 \mu \mathrm{L}$ of $1 \times$ Quenching Buffer and $4.5 \mu \mathrm{L}$ of sodium cyanoborohydride were added and incubated at room temperature for $15 \mathrm{~min}$ on a rotator. The liquid was removed from the column again by centrifuging. Finally, the resin was washed once with $300 \mu \mathrm{L}$ of $1 \times$ Coupling Buffer and 6 times with $200 \mu \mathrm{L}$ washing solution. When the immobilization was completed, the resin was mixed with $600 \mu \mathrm{L}$ of serum/plasma and gently rotated at $4{ }^{\circ} \mathrm{C}$ overnight. After that, $75 \mu \mathrm{L}$ elution buffer was added to the resin and incubated for $10 \mathrm{~min}$ at room temperature. IgE was collected by centrifuging at $1000 \mathrm{~g}$ for $1 \mathrm{~min}$.

\section{SDS-PAGE}

The eluate was lyophilized and analysed by Novex ${ }^{\circledR}$ NuPAGE ${ }^{\circledR}$ SDS-PAGE Gel System (Invitrogen Ltd, Paisley, UK). Samples were dissolved in NuPAGE ${ }^{\circledR}$ LDS Sample Buffer, incubated at $70{ }^{\circ} \mathrm{C}$ for $10 \mathrm{~min}$, loaded to Novex ${ }^{\circledR}$ NuPAGE $^{\circledR} 3-8 \%$ Tris-Acetate Mini Gels, and run at $150 \mathrm{~V}$ constant in Tris-Acetate SDS Running buffer. Gels were 
stained using Novex ${ }^{\circledR}$ Colloidal Blue Staining Kit. Gel bands of interest were chopped into $1 \times 1 \mathrm{~mm}$ pieces, which were destained at room temperature using $50 \mathrm{mM}$ ammonium hydrogen carbonate (Sigma-Aldrich, Poole, UK), pH 8.4 for 5 min, and mixed with equal amount of acetonitrile (Romil, Cambridge, UK) for another $5 \mathrm{~min}$. The supernatant was discarded. The gel pieces were completely destained by repeating the two steps several times and were dried on a Thermo Savant SPD121P Speed Vac (Thermo Scientific, Basingstoke, UK). The samples in the gel pieces were reduced using $10 \mathrm{mM}$ dithiothreitol (Roche Applied Science, East Sussex, UK) in ammonium hydrogen carbonate, $\mathrm{pH} 8.4$ at $56{ }^{\circ} \mathrm{C}$ for $30 \mathrm{~min}$. The dithiothreitol was then removed and the gel was washed and shrunk using $200 \mu \mathrm{L}$ acetonitrile, and dried in the Speed Vac. Reduced samples were carboxymethylated by adding $200 \mu \mathrm{L} 55$ mM iodoacetic acid (Sigma-Aldrich, Poole, UK)

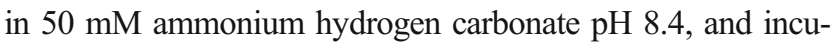
bating in the dark for $30 \mathrm{~min}$. After that, the iodoacetic acid solution was removed. The gel pieces were shrunk using $200 \mu \mathrm{L}$ acetonitrile, and dried in the Speed Vac.

\section{In-gel digestion and (glyco)peptides extraction}

The samples in the gel pieces were digested by trypsin (Promega, Hampshire, UK) or chymotrypsin (Promega, Hampshire, UK) into (glyco)peptides. Different proteolytic enzymes were utilized to facilitate analysis of individual glycosylation sites in glycopeptides. For trypsin digestion, 1/50 $(w / w)$ of trypsin in $50 \mathrm{mM}$ ammonium hydrogen carbonate, $\mathrm{pH} 8.4$ was added to the dried gel pieces and incubated for $37{ }^{\circ} \mathrm{C}$ overnight. For chymotrypsin digestion $1 / 20(w / w)$ of chymotrypsin in $50 \mathrm{mM}$ ammonium hydrogen carbonate, $\mathrm{pH} 7.8$ was added and incubated at $37{ }^{\circ} \mathrm{C}$ overnight. After the digestion, the supernatant was transferred to a new tube. Then $50 \mu \mathrm{L}$ of $0.1 \%(v / v)$ trifluoroacetic acid (Romil, Cambridge, UK) was added to the gel pieces and incubated at $37^{\circ} \mathrm{C}$ for $10 \mathrm{~min}$, followed by $100 \mu \mathrm{L}$ acetonitrile and incubated at $37^{\circ} \mathrm{C}$ for $15 \mathrm{~min}$. The supernatant was pooled with the previous supernatant. This step was repeated twice. The combined supernatant was reduced to $10 \mu \mathrm{L}$ in a Speed Vac for mass spectrometric analysis.

\section{Mass spectrometric analysis}

MALDI-TOF/TOF was used for protein identification of the gel bands. The (glyco)peptides were mixed with $\alpha$-CHCA (SigmaAldrich, Poole, UK) matrix $(10 \mathrm{mg} / \mathrm{mL}$ in $50 \%$ acetonitrile, $0.05 \%$ trifluoroacetic acid), spotted on a MALDI plate, dried, and analysed using a 4800 MALDI-TOF/TOFTM Analyzer (Applied Biosystems, Darmstadt, Germany). The data were analysed using GPS Explorer ${ }^{\mathrm{TM}}$ Software (Applied Biosystems, Darmstadt, Germany). Both MS and MS/MS data were used to search 283,454 entries in release 54.2 of the
SwissProt database with version 2.2 of the Mascot database search algorithm (www.matrixscience.com) with the following parameters: peptide masses were fixed as monoisotopic, partial oxidation of methionine residues was considered, partial carboxymethylation of cysteine residues was considered, the mass tolerance was set at $75 \mathrm{ppm}$ for precursor ions and $0.1 \mathrm{Da}$ for fragment ions and tryptic digests were assumed to have no more than one missed cleavage. Peptide matches from both MS and MS/MS data were used to generate probability-based Mowse protein scores. Scores of $>55$ were considered significant $(P<0.05)$. Glycoproteomic analysis was carried out according to a previous report [24], The (glyco) peptides were separated on a Pepmap C18 nanocapillary column (15 cm length, $75 \mathrm{~mm}$ internal diameter) fitted to a nano-HPLC system (LC Packings) connected to an ABI QSTAR Pulsar Hybrid LC/MS/MS system (Applied Biosystems/MDS Sciex). A gradient from $0.05 \%(v /$ v) formic acid in $95.5 \%(v / v)$ water/acetonitrile to $0.05 \%(v / v)$ formic acid in $95.5 \%(v / v)$ acetonitrile/water solution were employed. Data were analysed using Analyst QS Software.

\section{Results}

\section{Preparation and proteomic identification of IgE}

IgE from a patient with $P G M 3$ mutation and a patient with atopic dermatitis as a control subject was enriched by immunoprecipitation and analysed by non-reduced SDS-PAGE, which is shown in Fig. 1. There was a clear band with molecular weight between 160 and $220 \mathrm{kDa}$ in each sample, which is close to the predicted molecular weight of IgE $(190 \mathrm{kDa})$. Each band was digested by trypsin and identified as human $\operatorname{IgE}$ by proteomic MALDI-TOF/TOF analysis (Supplementary Table 1 and Supplementary Table 2). As detailed below, the two IgE bands were subjected to glycoproteomic analysis by on-line nano-LC-MS. In addition Supplementary Table 8-11 compile the nonglycosylated $\operatorname{IgE}$ peptides also characterised in this analysis.

\section{Glycoproteomic analysis of Asn21}

Glycosylation at Asn21 of the IgE from a patient with $P G M 3$ mutation is shown in Fig. 2. Glycopeptides generated by chymotryptic digestion ( ${ }^{12}$ TRCCKNIPSNATSVTL ${ }^{27}$ ) were detected eluting at three time points. Glycopeptides with nonsialylated glycans $\left(\mathrm{Hex}_{5} \mathrm{HexNAc}_{4-5} \mathrm{Fuc}\right)$ eluted between 30.5 and $31.5 \mathrm{~min}$, those with mostly mono-sialylated glycans $\left(\mathrm{NeuAc}_{0-1} \mathrm{Hex}_{5} \mathrm{HexNAc}_{4-5} \mathrm{Fuc}\right.$ ) eluted between from 32.5 to $33.5 \mathrm{~min}$, and those with mostly bi-sialylated glycans (NeuAc ${ }_{1-2} \mathrm{Hex}_{4-5} \mathrm{HexNAc}_{4-5} \mathrm{Fuc}$ ) eluted between 35 and $37 \mathrm{~min}$. All glycans were fucosylated and compositions 
IgE from a patient with allergy

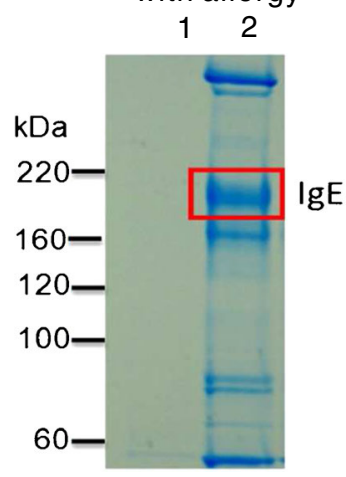

IgE from a patient with PGM3 mutation

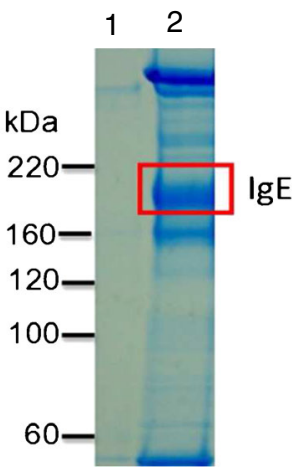

Fig. 1 SDS-PAGE of immunoprecipitated IgE. $600 \mathrm{ul}$ of serum from a patient with atopic dermatitis as a control subject and $600 \mathrm{ul}$ of plasma from a patient with hyper IgE syndrome were used to prepare $\operatorname{IgE}$. Elutions of immunoprecipitation were analyzed by non-reduced SDSPAGE in 3-8 \% Tris-acetate gel with Tris-acetate running buffer. Lane 1: Control resin. Lane 2: Immunoprecipitated IgE. A band was observed between 220 and $160 \mathrm{kDa}$ in Lane 2, which is consistent with the molecular weight of $\operatorname{IgE}$

consistent with bi-antennary structures dominated the spectra. The glycopeptides of the IgE from a control subject revealed a similar glycosylation profile (Table 1, Supplementary Figure 1, Supplementary Table 3).

\section{Glycoproteomic analysis of Asn49}

Glycosylation at Asn49 of the IgE from a patient with PGM3 mutation is shown in Fig. 3 (Table 1). Glycopeptides generated by chymotryptic digestion $\left({ }^{44}\right.$ DTGSLNGTTMTLPATTL $\left.{ }^{60}\right)$ were detected eluting at three time points. Glycopeptides with non-sialylated glycans $\left(\mathrm{Hex}_{5} \mathrm{HexNAc}_{4-5} \mathrm{Fuc}\right)$ eluted between 37 and $38 \mathrm{~min}$, those with mostly mono-sialylated glycans $\left(\mathrm{NeuAc}_{0-1} \mathrm{Hex}_{4-5} \mathrm{HexNAc}_{4-5} \mathrm{Fuc}\right.$ ) eluted between from 40 to $42 \mathrm{~min}$, and those with mostly bi-sialylated glycans $\left(\mathrm{NeuAc}_{1-}\right.$ ${ }_{2} \mathrm{Hex}_{4-5} \mathrm{HexNAc}_{4-5} \mathrm{Fuc}$ ) eluted between 46 and $47.5 \mathrm{~min}$. All glycans were fucosylated and compositions consistent with biantennary structures dominated the spectra. Again the glycopeptides of the IgE from a control subject revealed a similar
Fig. 2 Glycosylation of IgE Asn21 from a $P G M 3$ patient. Data were acquired by LC/MS.

The glycopeptides were produced by chymotrypsin digestion. The peptide backbone is presented as a black bar under each glycan $\left({ }^{12}\right.$ TRCCKNIPSNATSVTL ${ }^{27}$ ). The ions are in the form of $\mathrm{M}+$ $\mathrm{nH}^{\mathrm{n}+}$. Glycopeptides were detected eluting at three time points: from 30.5 to $31.5 \mathrm{~min}$ (a), from 32.5 to $33.5 \mathrm{~min}$ (b) and from 35 to $37 \mathrm{~min}(\mathbf{c})$. The glycan structures were deduced according to the molecular weight, fragmention of glycopeptides by MS/MS analysis, previous glycomic analysis of released $\operatorname{IgE} \mathrm{N}$ glycans and knowledge of $\mathrm{N}$ glycosylation biosynthetic pathways
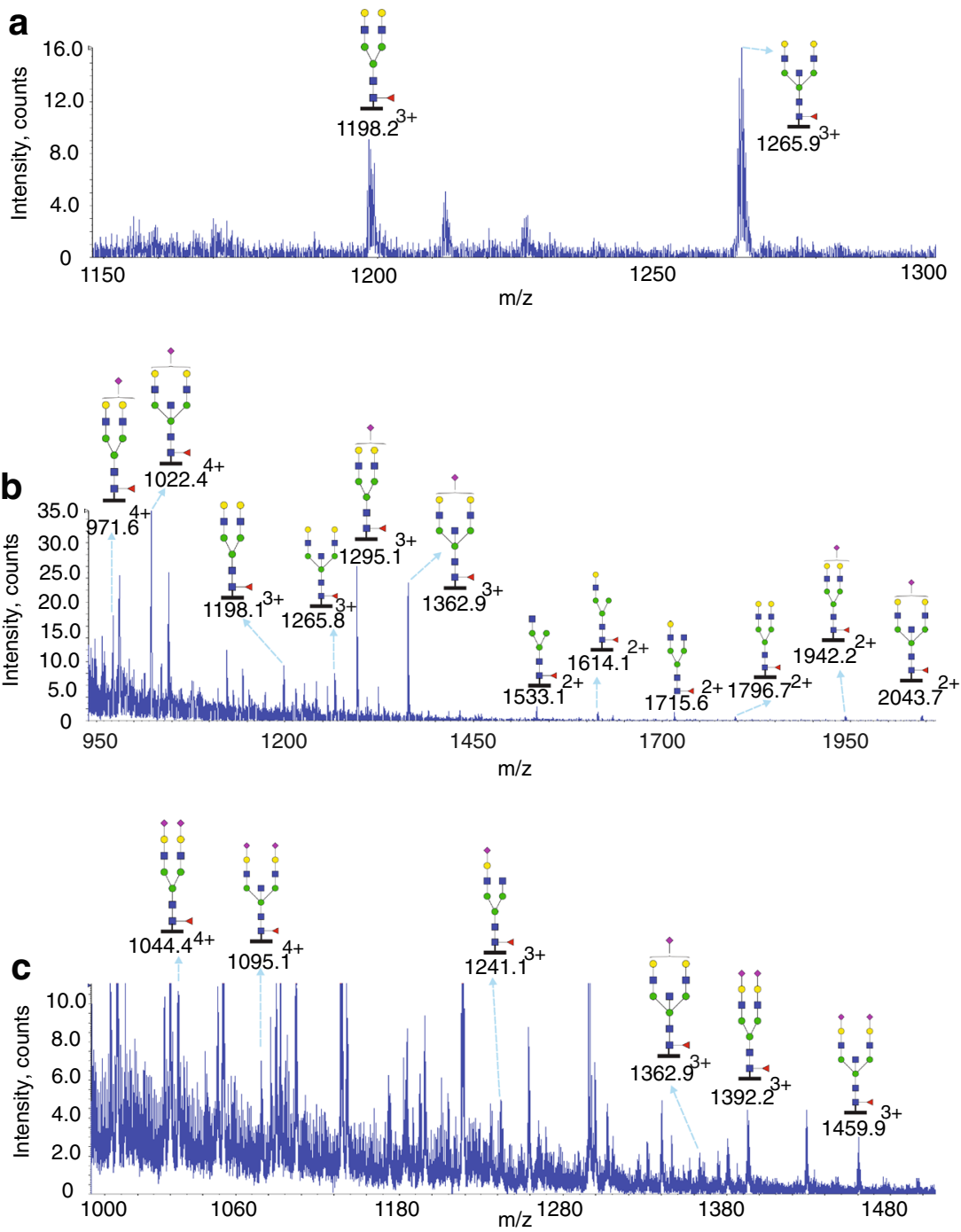
Table 1 Summary of distribution of glycan structures among the IgE glycosylation sites mapped using our glycoproteomic strategy. For detailed description see Supplementary Tables 3-7

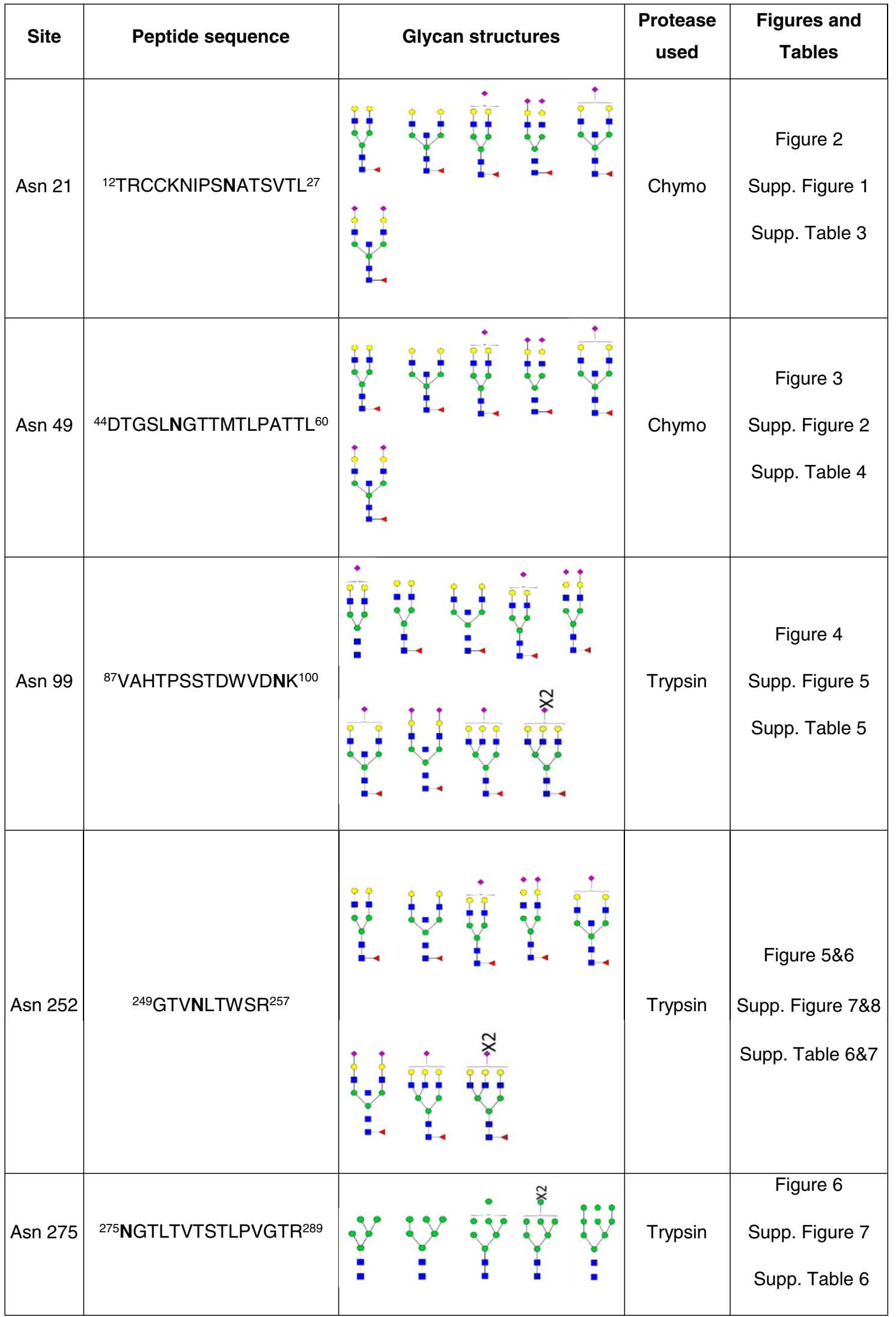


Fig. 3 Glycosylation of $\operatorname{IgE}$ Asn49 from a PGM3 patient. Data were acquired by LC/MS. The glycopeptides were produced by chymotrypsin digestion. The peptide backbone ( ${ }^{44}$ DTGSLNGTTMTLPATTL ${ }^{60}$ ) is presented as a black bar under each glycan. The ions are in the form of $\mathrm{M}+\mathrm{nH}^{\mathrm{n}+}$. Glycopeptides were detected eluting at three time points: from 37 to $38 \mathrm{~min}$ (a), from 40 to $42 \mathrm{~min}$ (b) and from 46 to $47.5 \mathrm{~min}$ (c). The glycan structures were deduced according to the molecular weight, fragmention of glycopeptides by MS/MS analysis, previous glycomic analysis of released IgE Nglycans and knowledge of Nglycosylation biosynthetic pathways
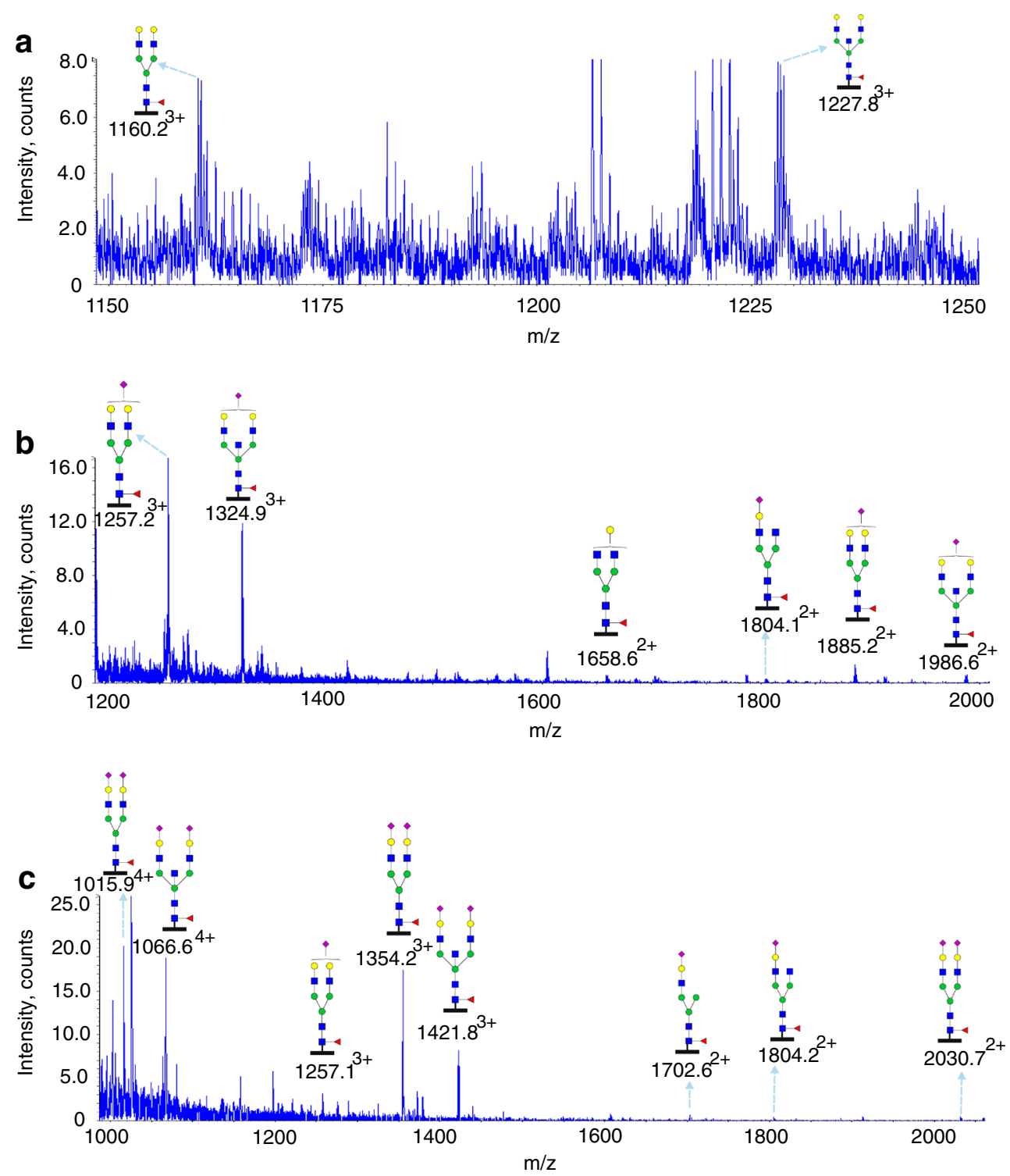

glycosylation profile (Table 1, Supplementary Figure 2, Supplementary Table 4). The N-glycosylation profiles of both IgE samples are very similar at Asn22 and Asn49 with nonsialylated, mono-sialylated and bi-sialylated glycopeptides.

To provide additional levels of structural definition MS/MS analysis was performed on selected molecular ions. Exemplary ESI-CID-MS/MS data of $\mathrm{m} / \mathrm{z} 1066.6^{4+}$ are shown in Supplementary Figure 3. The spectrum is dominated by glycan fragment ions $(\mathrm{m} / \mathrm{z} 168,186,204$ derived from GlcNAc; $m / z$ 256, 274 and 292 derived from sialic acid and $\mathrm{m} / \mathrm{z} 366$ derived from LacNAc). Moreover, two C-terminal peptide fragments were detected at $\mathrm{m} / \mathrm{z} 502$ and 615 . Collectively the data show that $\mathrm{m} / \mathrm{z} 1066.6^{4+}$ is the chymotryptic glycopeptide spanning Asn-49 carrying a core fucosylated, bisected, disialylated biantennary glycan.

\section{Glycoproteomic analysis of Asn99}

A greater diversity of $\mathrm{N}$-glycan structures was observed at Asn99 compared to Asn 21 and Asn49 in the two IgE samples. As indicated in Fig. 4 (Table 1 and Supplementary Table 5) glycopeptides generated by tryptic digestion $\left({ }^{87}\right.$ VAHTPSSTDWVDNK $\left.{ }^{100}\right)$ of IgE from a patient with $P G M 3$ mutation were detected eluting between 21 to 23 min. Compositionally the major N-glycans had biantennary structures with fucose and sialic acid, for example the two most abundant molecular ions at $\mathrm{m} / \mathrm{z} 1206.1^{3+}$ and $1303.2^{3+}$. A non-fucosylated glycan at $m / z 1157.4^{3+}$ was detected in both samples with low intensity. A small amount of non-sialylated glycans were detected, such as the peaks at $\mathrm{m} / \mathrm{z}$ $1109.1^{3+}$ and $m / z 1176.8^{2+}$. Potential tri-antennary glycans were observed at $m / z 996.2^{4+}, m / z 1327.9^{3+}$, and $1424.9^{3+}$ as 
Fig. 4 Glycosylation of IgE Asn99 from a $P G M 3$ patient. Data were acquired by LC/MS. The glycopeptides were produced by trypsin digestion. The peptide backbone

$\left({ }^{87}\right.$ VAHTPSSTDWVDNK $\left.{ }^{100}\right)$ is presented as a black bar under each glycan. The ions are in the form of $\mathrm{M}+\mathrm{nH}^{\mathrm{n}+}$. Glycopeptides were detected eluting between 21 and $23 \mathrm{~min}$. The glycan structures were deduced according to the molecular weight, fragmention of glycopeptides by MS/MS analysis, previous glycomic analysis of released $\mathrm{IgE} \mathrm{N}$ glycans and knowledge of $\mathrm{N}$ glycosylation biosynthetic pathways

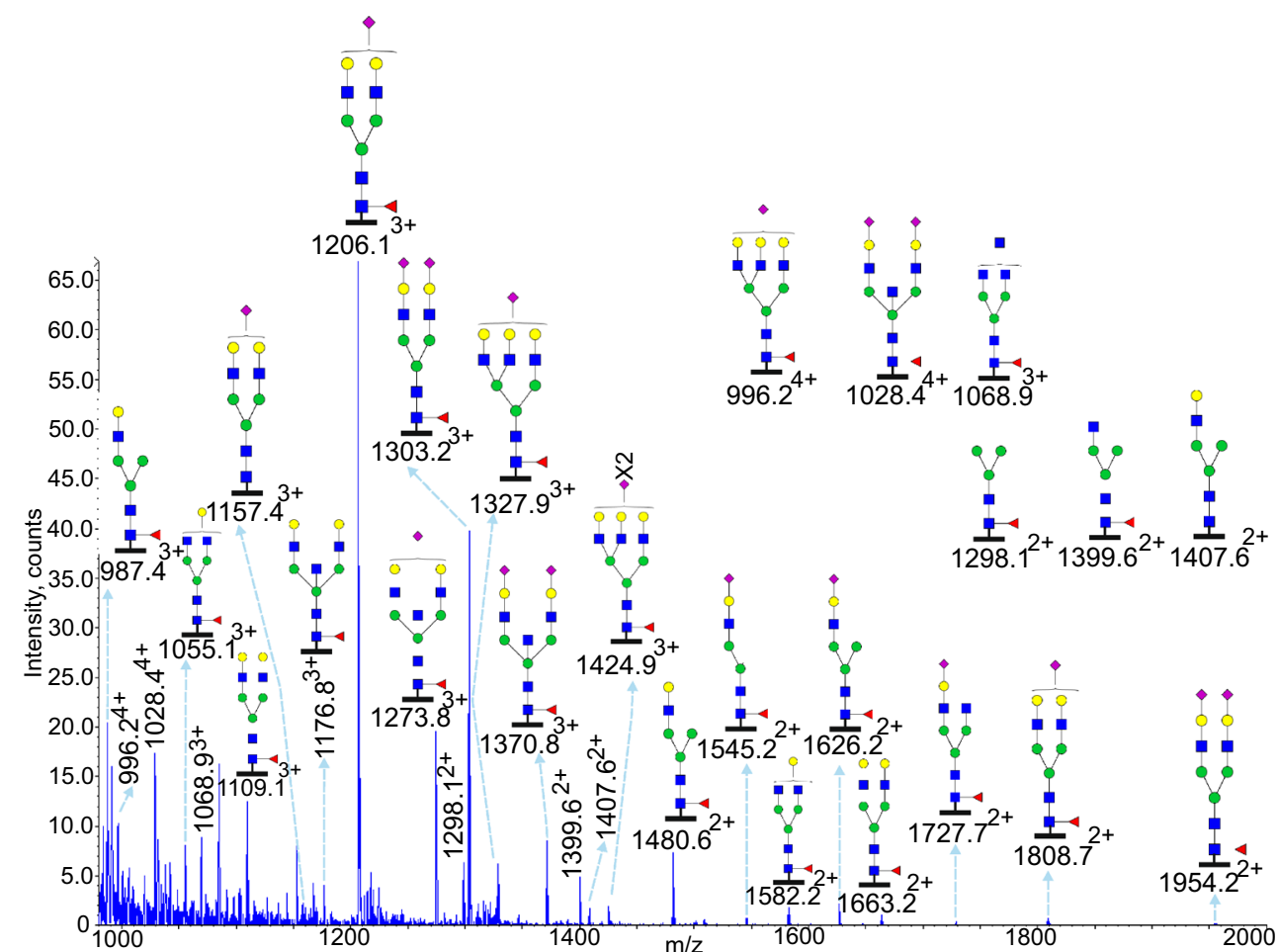

well as compositions consistent with mono-antennary complex structures such as $\mathrm{m} / \mathrm{z} 1480.6^{2+}, \mathrm{m} / \mathrm{z} 1545.2^{2+}$, and $1626.2^{2+}$. ESI-CID-MS/MS of the glycopeptide at $\mathrm{m} / \mathrm{z}$ $1206.1^{3+}$ is shown in Supplementary Figure 4. Similar to the fragment patterns observed in Supplementary Figure 3, glycan fragments at low $\mathrm{m} / \mathrm{z}$ range dominated the spectra. The peptide containing a GlcNAc residue $\left(\mathrm{m} / \mathrm{z} 880.4^{2+}\right.$ and $\left.\mathrm{m} / \mathrm{z} 1759.9^{+}\right)$ and the peptide with a GlcNAc and a Fuc residue $(\mathrm{m} / \mathrm{z}$ $1905.8^{+}$) provide strong evidence for the presence of core fucosylation. In addition, peptide y ions with glycan fragments were detected $\left(\mathrm{m} / \mathrm{z} 982.1^{2+}, 1063.0^{2+}, 1144.0^{2+}\right.$ and $\mathrm{m} / \mathrm{z} 1351.6^{+}$), which provides further information on both peptide sequence and glycan core structures. Again the glycopeptides of the IgE from a control subject revealed a similar glycosylation profile (Table 1, Supplementary Figure 5).

\section{Glycoproteomic analysis of Asn252 and Asn275}

Glycosylation at Asn252 of the IgE from a patient with $P G M 3$ mutation is shown in Figs. 5 and 6 (Table 1, Supplementary Table 6,7). Glycopeptides generated by tryptic digestion $\left({ }^{249}\right.$ GTVNLTWSR $\left.{ }^{257}\right)$ were detected eluting at two time points. At the first time point between 27.5 and 32 min glycopeptides containing Asn275 $\left({ }^{275}\right.$ NGTLTVTSTLPVGTR $\left.{ }^{289}\right)$ were also observed. Glycosylation at Asn252 $\left(\mathrm{NeuAc}_{0-1} \mathrm{Hex}_{4-6} \mathrm{HexNAc}_{4}\right.$ ${ }_{5} \mathrm{Fuc}_{1}$ ) contained mainly mono-sialylated bi-antennary glycans, such as $m / z 1099.4^{3+}$ and $m / z 1031.7^{3+}$, except for a tri-antennary glycan with a single sialic acid at $\mathrm{m} / \mathrm{z} 1153.5^{3+}$. ESI-CID-MS/ MS of $m / z 1002.4^{+}$of the PGM3 patient (Supplementary
Figure 6) confirmed the core fucosylated, non-sialylated glycan structure as annotated in the MS studies (Fig. 5). The second time point between $34.5 \mathrm{~min}$ and $36.5 \mathrm{~min}$, contained exclusively Asn252 containing glycopeptides. Mainly bi-sialyated glycans were observed $\left(\mathrm{NeuAc}_{0-2} \mathrm{Hex}_{3-5} \mathrm{HexNAc}_{3-5} \mathrm{Fuc}_{1}\right)$, as well as compositions consistent with mono-antennary complex structures such as $m / z 1364.6^{2+}$.

In contrast $\mathrm{N}$-glycans at Asn275 were high mannose glycans $\left(\mathrm{Hex}_{5-9} \mathrm{HexNAc}_{2}\right)$ and paucimannose glycans $\left(\mathrm{Hex}_{2-}\right.$ ${ }_{4} \mathrm{HexNAc}_{2}$ ) (Fig. 6, Table 1, Supplementary Table 7). Glycopeptides of the IgE from a control subject revealed a similar glycosylation profile (Table 1, Supplementary Figure 7, 8).

\section{Analysis of Asn264}

Glycosylation at Asn264 was not observed and the unglycosylated tryptic peptide $\left({ }^{258}\right.$ ASGKPVNHSTR $\left.{ }^{268}\right)$, was detected in both IgE samples (Supplementary Figure 9, Supplementary Figure 10) at $\mathrm{m} / \mathrm{z} 385.2^{3+}$ and $\mathrm{m} / \mathrm{z} 577.3^{2+}$. The MS/MS of $m / z 385.2^{3+}$ confirmed the peptide sequence (Supplementary figure 11).

\section{Discussion}

We previously demonstrated that the overall IgE N-glycan profiles were similar between a patient with $P G M 3$ mutation and patient with atopic dermatitis as a control subject [23]. In this paper we have now shown by glycoproteomic studies that 


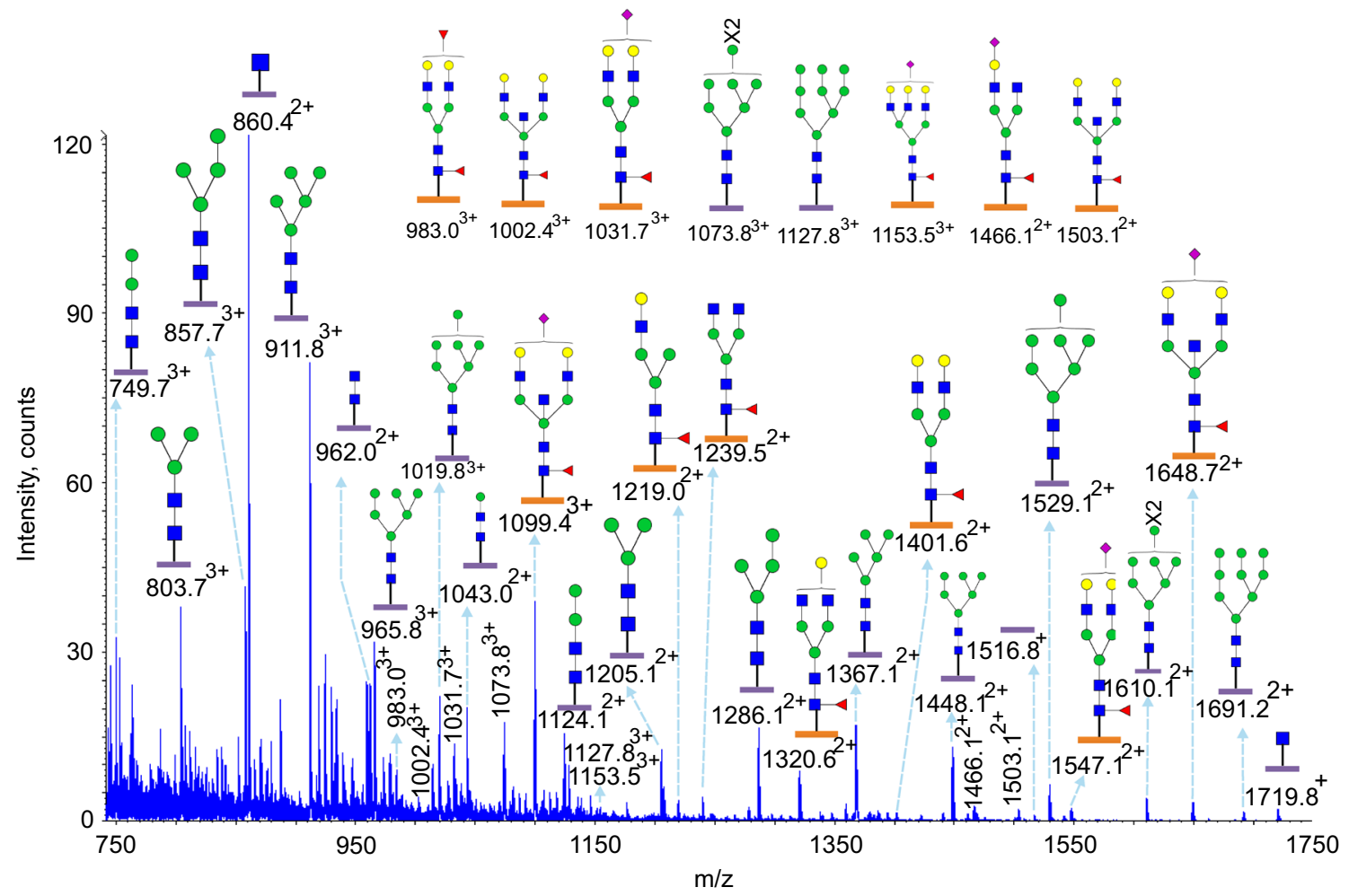

Fig. 5 Glycosylation of IgE Asn252 and Asn275 from a PGM3 patient. Data were acquired by LC/MS. The glycopeptides were produced by trypsin digestion. Peptide backbones with Asn252 $\left({ }^{249}\right.$ GTVNLTWSR $\left.^{257}\right)$ and Asn275 $\left({ }^{275}\right.$ NGTLTVTSTLPVGTR $\left.{ }^{289}\right)$ are presented as an orange bar and a purple bar respectively under each glycan. The ions are in the form of $\mathrm{M}+\mathrm{nH}^{\mathrm{n}+}$. Glycopeptides were eluted between 27.5 and $32 \mathrm{~min}$. The glycan structures were deduced according to the molecular weight, fragmention of glycopeptides by MS/MS analysis, previous glycomic analysis of released $\operatorname{IgE} \mathrm{N}$-glycans and knowledge of $\mathrm{N}$-glycosylation biosynthetic pathways

demonstrate that $\operatorname{IgE}$ glycosylation is similar among healthy controls, allergy patients and patients with PGM3 related
Fig. 6 Glycosylation of $\operatorname{IgE}$ Asn252 from a $P G M 3$ patient. Data were acquired by LC/MS. The glycopeptides were produced by trypsin digestion. The peptide backbone with $\left({ }^{249}\right.$ GTVNLTWSR $\left.^{257}\right)$ is presented as an orange bar under each glycan. The ions are in the form of $\mathrm{M}+\mathrm{nH}^{\mathrm{n}+}$. The glycopeptides were eluted from 34.5 to $36.5 \mathrm{~min}$. The glycan structures were deduced according to the molecular weight, fragmention of glycopeptides by MS/MS analysis, previous glycomic analysis of released IgE Nglycans and knowledge of Nglycosylation biosynthetic pathways

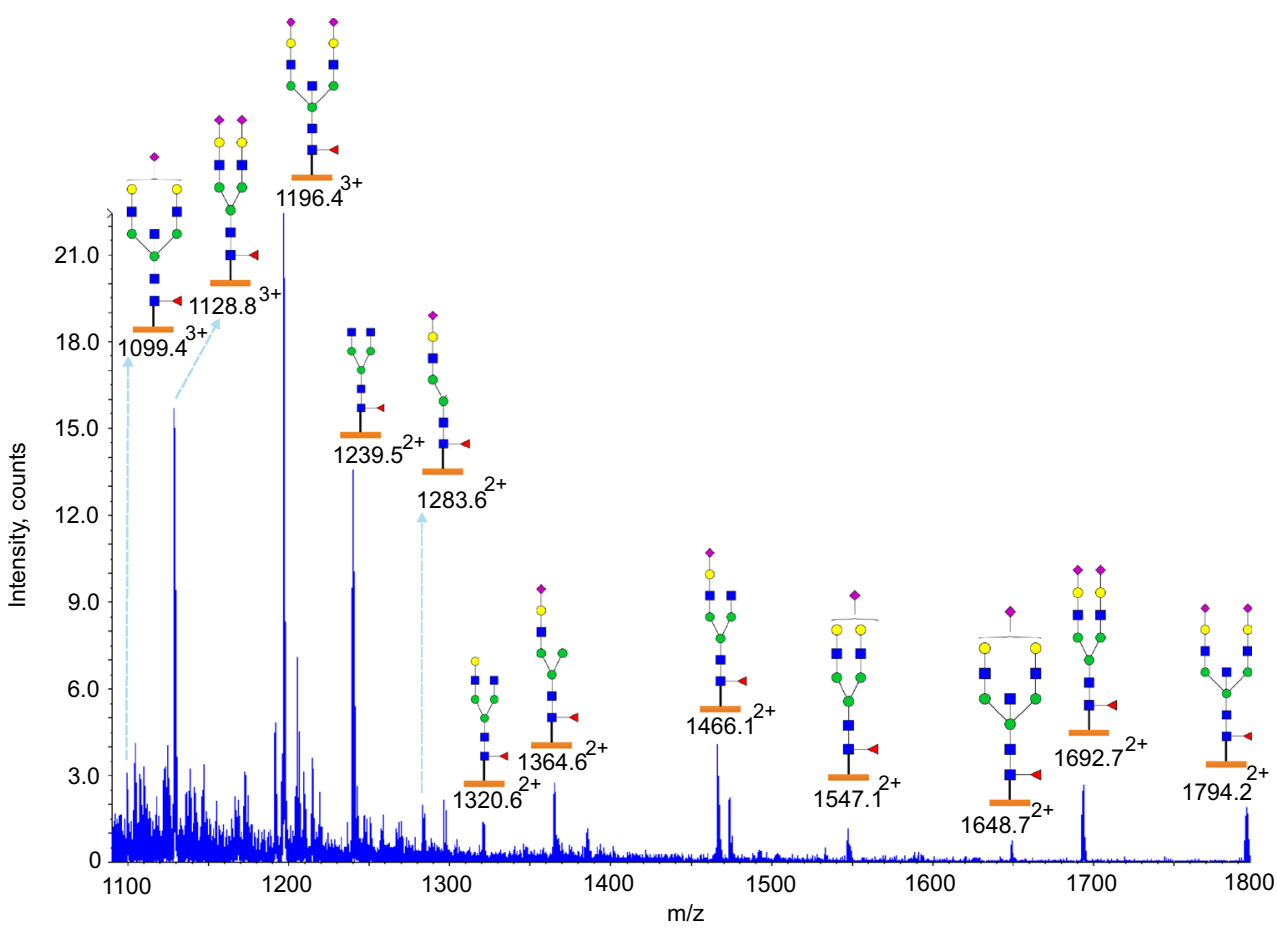


hyper IgE syndrome. Our glycoproteomic studies found that Asn264 was not occupied by N-glycans and Asn275 had only high mannose glycans, which is consistent with all previous site specific glycosylation studies [10-12, 14]. A trace amount of Fuc was detected in an myeloma IgE at Asn99 reported in 1970s [10-12], which is in contrast to the latest glycoproteomic report [14]. Our studies again found the glycans were mostly core fucosylated at this site. A HPLC based glycomic analysis [13] of an allergic IgE indicated that all the seven potential $\mathrm{N}$-glycosylation sites were glycosylated, while our results together with previous studies [10-12] and the most recent report [14] showed Asn 264 is not glycosylated. Asn146 was not mapped in this study, which coincides with a previous report that this site cannot be mapped by trypsin or chymotrypsin digestion [14]. Nevertheless, despite not characterising glycosylation at Asn146 we still conclude that IgE glycosylation does not change in allergy and $P G M 3$ related hyper IgE syndrome, because we have both the overall glycan profile of IgE and glycoproteomic data from 6 of the 7 glycosylation sites in the glycoproteomic data. Combining the two sets of data, we feel it is highly unlikely that the glycosylation at Asn146 does change.

Both glycomic [23] and glycoproteomic studies showed $P G M 3$ mutation did not affect IgE glycosylation, although elevated IgE is a key symptom in these patients. A possible explanation is that the major complex N-glyans on IgE have biantennary structures. Our previous studies of B cells and neutrophils from $P G M 3$ mutation patients have shown that tri-/tetraantennary glycans are most sensitive to biochemical changes caused by $P G M 3$ mutation, while bi-antennary glycans are not as sensitive [23]. The elevated IgE may be caused by decreased tri-/tetra-antennary glycans on other glycoproteins that are involved in $\operatorname{IgE}$ production. In addition, it has been known that O-GlcNAcylation is hypersensitive to UDP-GlcNAc concentration [25]. A mouse model with hypomorphic mutations in Pgm3 has shown decreased O-GlcNAcylation [26]. Therefore, impairment of O-GlcNAcylation could be another cause of the elevated $\operatorname{IgE}$ in the patients.

Acknowledgments This work was also supported by the Biotechnology and Biological Sciences Research Council (BBF0083091 and BB/ K016164/1 to A.D., HRM and S.M.H). G.W. is grateful for a Sun Hung Kai Properties-Kwoks' Foundation-Imperial College PhD Scholarship.

Open Access This article is distributed under the terms of the Creative Commons Attribution 4.0 International License (http:// creativecommons.org/licenses/by/4.0/), which permits unrestricted use, distribution, and reproduction in any medium, provided you give appropriate credit to the original author(s) and the source, provide a link to the Creative Commons license, and indicate if changes were made.

\section{References}

1. Arnold, J.N., Wormald, M.R., Sim, R.B., Rudd, P.M., Dwek, R.A.: The impact of glycosylation on the biological function and structure of human immunoglobulins. Annu. Rev. Immunol. 25, 21-50 (2007)

2. Parekh, R.B., Dwek, R.A., Sutton, B.J., Fernandes, D.L., Leung, A., Stanworth, D., Rademacher, T.W., Mizuochi, T., Taniguchi, T., Matsuta, K., et al.: Association of rheumatoid arthritis and primary osteoarthritis with changes in the glycosylation pattern of total serum IgG. Nature 316(6027), 452-457 (1985)

3. Anthony, R.M., Wermeling, F., Ravetch, J.V.: Novel roles for the IgG Fc glycan. Ann. N. Y. Acad. Sci. 1253, 170-180 (2012)

4. Scallon, B.J., Tam, S.H., McCarthy, S.G., Cal, A.N., Raju, T.S.: Higher levels of sialylated Fc glycans in immunoglobulin G molecules can adversely impact functionality. Mol. Immunol. 44(7), 1524-1534 (2007)

5. Anthony, R.M., Wermeling, F., Karlsson, M.C., Ravetch, J.V.: Identification of a receptor required for the anti-inflammatory activity of IVIG. Proc. Natl. Acad. Sci. U. S. A. 105(50), 1957119578 (2008)

6. Royle, L., Roos, A., Harvey, D.J., Wormald, M.R., van Gijlswijk-Janssen, D., Redwan, E.M., Wilson, I.A., Daha, M.R., Dwek, R.A., Rudd, P.M.: Secretory $\operatorname{IgA}$ N- and Oglycans provide a link between the innate and adaptive immune systems. J. Biol. Chem. 278(22), 20140-20153 (2003)

7. Basset, C., Devauchelle, V., Durand, V., Jamin, C., Pennec, Y.L., Youinou, P., Dueymes, M.: Glycosylation of immunoglobulin A influences its receptor binding. Scand. J. Immunol. 50(6), 572579 (1999)

8. Goetze, A.M., Liu, Y.D., Zhang, Z., Shah, B., Lee, E., Bondarenko, P.V., Flynn, G.C.: High-mannose glycans on the Fc region of therapeutic $\operatorname{IgG}$ antibodies increase serum clearance in humans. Glycobiology 21(7), 949-959 (2011)

9. Gould, H.J., Sutton, B.J.: IgE in allergy and asthma today. Nat. Rev. Immunol. 8(3), 205-217 (2008)

10. Dorrington, K.J., Bennich, H.H.: Structure-function relationships in human immunoglobulin E. Immunol. Rev. 41, 3-25 (1978)

11. Baenziger, J., Kornfeld, S., Kochwa, S.: Structure of the carbohydrate units of IgE immunoglobulin. II. Sequence of the sialic acidcontaining glycopeptides. J. Biol. Chem. 249(6), 1897-1903 (1974)

12. Baenziger, J., Kornfeld, S., Kochwa, S.: Structure of the carbohydrate units of IgE immunoglobulin. I. Over-all composition, glycopeptide isolation, and structure of the high mannose oligosaccharide unit. J. Biol. Chem. 249(6), 1889-1896 (1974)

13. Arnold, J.N., Radcliffe, C.M., Wormald, M.R., Royle, L., Harvey, D.J., Crispin, M., Dwek, R.A., Sim, R.B., Rudd, P.M.: The glycosylation of human serum $\operatorname{IgD}$ and $\operatorname{IgE}$ and the accessibility of identified oligomannose structures for interaction with mannan-binding lectin. J. Immunol. 173(11), 6831-6840 (2004)

14. Plomp, R., Hensbergen, P.J., Rombouts, Y., Zauner, G., Dragan, I., Koeleman, C.A., Deelder, A.M., Wuhrer, M.: Site-specific N-glycosylation analysis of human immunoglobulin e. J. Proteome Res. 13(2), 536-546 (2014)

15. Yong, P.F.K., Freeman, A.F., Engelhardt, K.R., Holland, S., Puck, J.M., Grimbacher, B.: An update on the hyper-IgE syndromes. Arthritis Res. Ther. 14(6), 228 (2012)

16. Davis, S.D., Schaller, J., Wedgwood, R.J.: Job's Syndrome. Recurrent, "cold", staphylococcal abscesses. Lancet 1, 1013 1015 (1966)

17. Grimbacher, B., Holland, S.M., Gallin, J.I., Greenberg, F., Hill, S.C., Malech, H.L., Miller, J.A., O'Connell, A.C., Puck, J.M.: Hyper-IgE syndrome with recurrent infections - an autosomal dominant multisystem disorder. N. Engl. J. Med. 340(9), 692-702 (1999) 
18. Holland, S.M., DeLeo, F.R., Elloumi, H.Z., Hsu, A.P., Uzel, G., Brodsky, N., Freeman, A.F., Demidowich, A., Davis, J., Turner, M.L., Anderson, V.L., Darnell, D.N., Welch, P.A., Kuhns, D.B., Frucht, D.M., Malech, H.L., Gallin, J.I., Kobayashi, S.D., Whitney, A.R., Voyich, J.M., Musser, J.M., Woellner, C., Schaffer, A.A., Puck, J.M., Grimbacher, B.: STAT3 mutations in the hyper-IgE syndrome. N. Engl. J. Med. 357(16), 1608-1619 (2007)

19. Minegishi, Y., Saito, M., Tsuchiya, S., Tsuge, I., Takada, H., Hara, T., Kawamura, N., Ariga, T., Pasic, S., Stojkovic, O., Metin, A., Karasuyama, H.: Dominant-negative mutations in the DNAbinding domain of STAT3 cause hyper-IgE syndrome. Nature 448(7157), 1058-1062 (2007)

20. Zhang, Q.A., Davis, J.C., Dove, C.G., Su, H.C.: Genetic, clinical, and laboratory markers for DOCK8 immunodeficiency syndrome. Dis. Markers 29(3-4), 131-139 (2010)

21. Engelhardt, K.R., McGhee, S., Winkler, S., Sassi, A., Woellner, C., Lopez-Herrera, G., Chen, A., Kim, H.S., Lloret, M.G., Schulze, I., Ehl, S., Thiel, J., Pfeifer, D., Veelken, H., Niehues, T., Siepermann, K., Weinspach, S., Reisli, I., Keles, S., Genel, F., Kutuculer, N., Camcioglu, Y., Somer, A., Karakoc-Aydiner, E., Barlan, I., Gennery, A., Metin, A., Degerliyurt, A., Pietrogrande, M.C., Yeganeh, M., Baz, Z., Al-Tamemi, S., Klein, C., Puck, J.M., Holland, S.M., McCabe, E.R.B., Grimbacher, B., Chatila, T.A.: Large deletions and point mutations involving the dedicator of cytokinesis 8 (DOCK8) in the autosomal-recessive form of hyper-IgE syndrome. J. Allergy Clin. Immunol. 124(6), 1289-1302 (2009)

22. Zhang, Y., Yu, X., Ichikawa, M., Lyons, J.J., Datta, S., Lamborn, I.T., Jing, H., Kim, E.S., Biancalana, M., Wolfe, L.A., DiMaggio, T., Matthews, H.F., Kranick, S.M., Stone, K.D., Holland, S.M., Reich, D.S., Hughes, J.D., Mehmet, H., McElwee, J., Freeman, A.F., Freeze, H.H., Su, H.C., Milner, J.D.: Autosomal recessive phosphoglucomutase 3 (PGM3) mutations link glycosylation defects to atopy, immune deficiency, autoimmunity, and neurocognitive impairment. J. Allergy Clin. Immunol. 133(5), 1400-1409 (2014). 1409 e1401-1405

23. Sassi, A., Lazaroski, S., Wu, G., Haslam, S.M., Fliegauf, M., Mellouli, F., Patiroglu, T., Unal, E., Ozdemir, M.A., Jouhadi, Z., Khadir, K., Ben-Khemis, L., Ben-Ali, M., Ben-Mustapha, I., Borchani, L., Pfeifer, D., Jakob, T., Khemiri, M., Asplund, A.C., Gustafsson, M.O., Lundin, K.E., Falk-Sorqvist, E., Moens, L.N., Gungor, H.E., Engelhardt, K.R., Dziadzio, M., Stauss, H., Fleckenstein, B., Meier, R., Prayitno, K., Maul-Pavicic, A., Schaffer, S., Rakhmanov, M., Henneke, P., Kraus, H., Eibel, H., Kolsch, U., Nadifi, S., Nilsson, M., Bejaoui, M., Schaffer, A.A., Smith, C.I., Dell, A., Barbouche, M.R., Grimbacher, B.: Hypomorphic homozygous mutations in phosphoglucomutase 3 (PGM3) impair immunity and increase serum IgE levels. J. Allergy Clin. Immunol. 133(5), 1410-1419 (2014). 1419 e1411-1413

24. Harrison, R., Hitchen, P.G., Panico, M., Morris, H.R., Mekhaiel, D., Pleass, R.J., Dell, A., Hewitt, J.E., Haslam, S.M.: Glycoproteomic characterization of recombinant mouse alpha-dystroglycan. Glycobiology 22(5), 662-675 (2012)

25. Hart, G.W., Slawson, C., Ramirez-Correa, G., Lagerlof, O.: Cross talk between O-GlcNAcylation and phosphorylation: roles in signaling, transcription, and chronic disease. Annu. Rev. Biochem. 80(80), 825-858 (2011)

26. Greig, K.T., Antonchuk, J., Metcalf, D., Morgan, P.O., Krebs, D.L., Zhang, J.G., Hacking, D.F., Bode, L., Robb, L., Kranz, C., de Graaf, C., Bahlo, M., Nicola, N.A., Nutt, S.L., Freeze, H.H., Alexander, W.S., Hilton, D.J., Kile, B.T.: Agm1/Pgm-3-mediated sugar nucleotide synthesis is essential for hematopoiesis and development. Mol. Cell. Biol. 27(16), 5849-5859 (2007) 